\title{
The impact of frailty on trauma outcomes using the Clinical Frailty Scale
}

\author{
Amari Thompson $^{1}$ (1) $\cdot$ Sunil Gida ${ }^{1} \cdot$ Yasar Nassif $^{1} \cdot$ Carla Hope $^{2} \cdot$ Adam Brooks $^{1}$
}

Received: 7 November 2020 / Accepted: 21 February 2021 / Published online: 8 March 2021

(c) Crown 2021

\begin{abstract}
Background Population ageing is a worldwide phenomenon; thanks to improvements in medical care and living standards. The Office of National Statistics in the UK predicts that the fastest growing age group in coming decades will be those over 85 years. This is reflected in Trauma Audit and Research Network data, which has highlighted a shift in caseload from a majority of young males to elderly patients at UK Major Trauma Centres (MTC). This study of elderly trauma patients admitted to a UK MTC reviews the links between frailty, using the Canadian Study of Health and Aging Clinical Frailty Scale (CFS), and outcomes from trauma.

Methods A retrospective database review of patients $>65$ years old admitted to our MTC was performed. We identified 1125 eligible patients of which 729 had a recorded CFS. Those without a CFS were omitted. The primary outcome measured was in-hospital mortality. Secondary measures were Injury Severity Score, length of stay, trauma team activation on arrival and discharge destination. Multivariate regression analyses were performed using STATA v 15.

Results Those of CFS 5-9 (frail) were 2.6 times more likely to die than the CFS 1-4 (pre-frail) (OR 2.65, 95\% CI 1.47-4.78). The frail group was also $56 \%$ less likely to have a trauma call on admission (OR 0.44, 95\% CI 0.30-0.65) and 61\% less likely to be discharged to their usual place of residence (OR 0.39, 95\% CI 0.28-0.55).

Conclusion We advocate the use of the Clinical Frailty Scale as a screening tool for frailty in trauma patients, highlighting those at risk of increased length of stay and mortality, subsequently assisting healthcare providers with setting realistic expectations with family members.
\end{abstract}

Level of evidence Level III, prognostic and epidemiological

Keywords Geriatric trauma $\cdot$ Elderly trauma $\cdot$ Frailty screening $\cdot$ Major trauma

The preliminary findings of this study were to be presented as a poster at the 20th European Congress of Trauma and Emergency Surgery 2020. However, due to COVID-19 the event was cancelled but the abstract has been published.

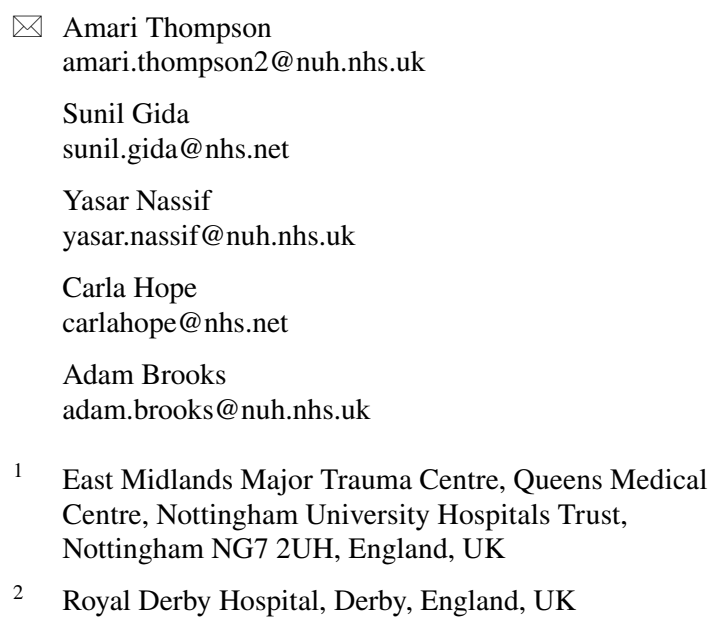

2 Royal Derby Hospital, Derby, England, UK

\section{Background}

In 2016, there were 11.8 million residents aged 65 and older, representing $18 \%$ of the UK population [1]. By 2066, it is anticipated that this population will almost double to 20.4 million, $26 \%$ of the total population [1]. Additionally, it is predicted that the fastest increase will be seen in those $>85$ years. In recent years, the Trauma Audit and Research Network (TARN) has noted that elderly patients falling less than $2 \mathrm{~m}$ have become the most common trauma admission [2]. This is multi-factorial as awareness of geriatric trauma spreads alongside the availability of diagnostic CT. With these two factors in mind, it is likely geriatric trauma will continue to contribute a significant proportion of major trauma workload.

When caring for elderly patients, it is important to consider their differing needs compared to the general adult population. A statement from the Frailty Consensus 
Conference in 2012 defined frailty as "a medical syndrome with multiple causes and contributors that is characterised by diminished strength, endurance and reduces physiological function that increases an individual's vulnerability for developing increased dependency and/or death" [3]. Clearly frail patients suffering from trauma need to be identified early, to enable clinicians to make appropriate decisions regarding their ongoing care. A large systematic review has found physical frailty to be present in $9.6-10.2 \%$ (mean $9.9 \%$ ) of participants aged greater than 65 . This proportion only increased when psychosocial frailty was included [4]. As such, it is vital to review frailty alongside trauma outcomes; not to do this neglects the aforementioned findings.

As elderly trauma admissions are likely to continue to rise, then practice at Major Trauma Centres (MTC) must adapt. The British Geriatric Society recommends that frailty assessments are performed for all elderly patients at any interaction with a healthcare professional [5]. At the East Midlands Major Trauma Centre, Nottingham, we have used the Canadian Study of Health and Aging (CSHA) Clinical Frailty Scale (CFS) to identify frail patients since April 2019 [6]. The CFS is a tool easily used by clinicians, which can help to identify levels of frailty, predict institutionalisation and death [6].

The Clinical Frailty Scale is an operational classification validated during the third stage of the CSHA. At the

\section{Clinical Frailty Scale*}

(

Very Fit - People who are robust, active, energetic and motivated. These people commonly exercise regularly. They are among the fittest for their age.

1

2 Well-People who have no active disease symptoms but are less fit than category I. Often, they exercise or are very active occasionally, e.g. seasonally.

3 Managing Well - People whose medical problems are well controlled, but are not regularly active beyond routine walking.

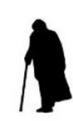

4 Vulnerable - While not dependent on others for daily help, often symptoms limit activities. A common complaint is being "slowed up", and/or being tired during the day.

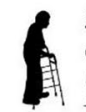

5 Mildly Frail - These people often have more evident slowing, and need help in high order IADLs (finances, transportation, heavy housework, medications). Typically, mild frailty progressively impairs shopping and walking outside alone, meal preparation and housework.

6 Moderately Frail - People need help with all outside activities and with keeping house. Inside, they often have problems with stairs and need help with bathing and might need minimal assistance (cuing, standby) with dressing. end of CSHA-2 in 1996, participants were interviewed by a clinician and allocated a score on the CFS; this was then reviewed by a multidisciplinary team of which the initial clinician was part of. After 5 years, the cohort was followed up and the CFS was compared with the CSHA Frailty Index for validity. The CSHA frailty index being a mathematically derived score taking into account 70 variables. There was found to be high correlation between the two scores (Pearson coefficient $0.8 p<0.01$ ) [6].

The scale is from 1 to 9 (Fig. 1). Each point on the scale has overall summary of the patient's condition and a title, e.g. "1 very fit", "2 managing well" and "7 severely frail", etc. Additional information is provided on scoring frailty in the presence of dementia. The current scale was revised in 2007 following the original publication; it now includes two additional groups being " 8 very severely frail" and "9 terminally ill".

The aim of this study is investigate the impact of frailty, measured using CFS, on outcomes in trauma. We hypothesised that with increasing frailty, age and injury severity score (ISS) comes increased length of hospital stay and inhospital mortality. Once the most at-risk populations have been identified, further analysis to find factors predicting worse outcomes can be discovered.

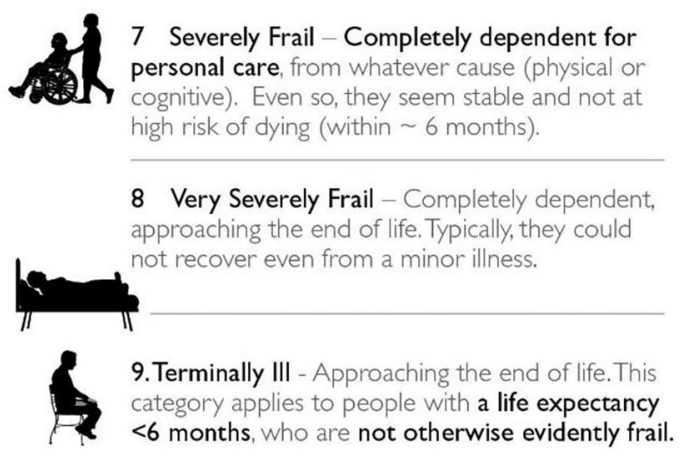

Scoring frailty in people with dementia

The degree of frailty corresponds to the degree of dementia. Common symptoms in mild dementia include forgetting the details of a recent event, though still remembering the event itself, repeating the same question/story and social withdrawal.

In moderate dementia, recent memory is very impaired, even though they seemingly can remember their past life events well. They can do personal care with prompting.

In severe dementia, they cannot do personal care without help.

* I. Canadian Study on Health \& Aging, Revised 2008. 2. K. Rockwood et al. A global clinical measure of fitness and frailty in elderly people. CMAJ 2005; 173:489-495. Q 2009. Version 1.2_EN. All rights reserved, Geriarric Medicine Research, Dahouse University, Haifinx, Ganadi Fermission granted

Fig. 1 The Clinical Frailty Scale 


\section{Methods}

Patients over the age of 65 years admitted to a single UK MTC (Queen's Medical Centre, Nottingham) over 1 year (April 2019-April2020) were retrospectively reviewed. Patient data were extracted from data submitted to the Trauma Audit and Research Network by the hospital. Predictor variables included age, sex, Injury Severity Score (ISS), mechanism of injury and CFS. CFS scores were allocated to patients following review in Accident and Emergency by clinician. A proportion of those admitted patients would have been reviewed by either a consultant or registrar in Geriatric Medicine, producing a final CFS score. CFS was divided into two groups, CFS 1-4 classed as pre-frail and CFS 5-9 frail. Age and ISS were treated as continuous variables. The primary outcome measure was death during index admission. Secondary outcomes were length of stay, trauma team activation and discharge destination. Trauma team activation defined as a trauma alert being raised on arrival to the Emergency Department according to local protocol. Discharge destination was categorised as either discharged to usual place of residence or discharged to non-usual place of residence (e.g. care home, residential home or hospice).

Continuous data are presented as medians with interquartile range (IQR). Variables were checked for normal distribution using histograms. Group differences for continuous variables were performed using independent $t$ test or Wilcoxon rank test as appropriate. Categorical data are reported as proportions and tested for significance using Chi-square test.

Univariate logistic and linear regressions were performed. Odds ratios, $\beta$ value and $95 \%$ confidence intervals were reported as appropriate. In the multiple regressions, age and sex were considered a priori confounders. Multiple logistic and linear regressions were performed using pre-selected clinically relevant variables. $p$ values $<0.05$ were taken as significant. All analyses were performed using STATA version 15 (StataCorp LLC, College Station, TX, USA).

\section{Results}

In total, 1125 patients over the age of 65 were admitted during the time period. 396 (35.2\%) did not have a documented CFS and were omitted from the analysis. 729 patients were included in the analysis. 275 (37.7\%) were men and 454 women with a median age of 84 years (IQR 79-89). Fall less than $2 \mathrm{~m}$ was the most common cause of injury in this patient group (84.2\%). 81.7\% had a GCS of 15 on admission. $52 \%$ of CFS scores were allocated by Geriatrician. $60.9 \%$
(444/729) had a CFS of 5-9. The median ISS was 10 (IQR 9-20). The median total LOS was 11 days (IQR 7-18). Only $5.6 \%$ were admitted to intensive care at any point during admission, the length of intensive care stay ranged from 0 to 50 days. The overall mortality rate was $9.9 \%$.

There were few differences between the male and female group. Average age was 4 years younger in the male group in comparison to female ( 81 years IQR $77-87$ vs 85 years IQR 81-90). Average ISS was higher in male group (16 vs 9) as too was mortality, with $13.5 \%$ dying compared to 7.7\%. Mean admission GCS, Charleson comorbidity score and median total LOS were equivocal between the genders. Female participants were more likely to have a trauma call activated on admission (86.6 vs $74.9 \%$ ) and less likely to be to be discharged to their usual residence ( 49.3 vs $58.6 \%$ ).

The differences in CFS group 1-4 and CFS group 5-9 are summarised in Table 1. There were significant differences in gender, age, ISS, Charleson comorbidity score, discharge destination, trauma call likelihood, and death between the groups $(p<0.05)$. Those in CFS group $1-4$ were significantly younger than those in CFS group 5-9 (Mean dif $=-4.44, t(727)=-8.40, p<0.001)$. The ISS was significantly lower in CFS group 5-9 than CFS group $1-4(Z=2.282, p=0.00225)$. Those in CFS group 5-9 had a higher Charleson comorbidity score (mean dif $=-0.83$, $t(727)=-7.43, p<0.001)$. Those in CFS group $1-4$ were more likely have a trauma call $(25.3$ vs $13.15, p<0.001)$. Those in CFS group 1-4 were significantly more likely to be discharged to their usual residence than those with CFS $5-9$ (71.5 vs $49.6 \%, p<0.001)$. Those in CFS group $1-4$ were less likely to die (5.3 vs $12.8 \%, p<0.001$ ).

In univariate analysis, CFS was significantly associated with trauma call likelihood, discharge destination, length of stay and mortality (Table 2). Those with a CFS score 5-9 were 56\% less likely to have a trauma call at time of admission (OR 0.44, 95\% CI 0.30-0.65). Those with CFS 5-9 were $61 \%$ less likely to be discharged to their usual place of residence (OR 0.39, 95\% CI 0.28-0.55). Those with CFS 5-9, stayed on average 2 days longer than those with CFS $1-4$ ( $\beta 2.17,95 \%$ CI $0.25-4.09)$. Those in CFS group 5-9 were 2.6 times more likely to die than those in CFS group 1-4 (OR 2.65, 95\% CI 1.47-4.78).

After adjustment, CFS was an independent predictor of length of stay, discharge destination and mortality (Table 2). CFS remained an independent predictor of length of stay after adjustment (OR 2.08, 95\% CI 0.03-4.12). Those in CFS group 5-9 were 56\% less likely to be discharged to their usual residence (OR $0.44,95 \%$ CI 0.31-0.63), after taking into account other factors. After adjusting for age, sex, Charleson comorbidity index, ISS, trauma call status and length of stay, those in CFS group 5-9 remained more than twice as likely to die as those in CFS group 1-4 (OR 2.79, 95\% CI 1.47-5.28). However, 
Table 1 Summary of differences between CFS group $1-4$ and CFS group 5-9

\begin{tabular}{lllc}
\hline & CFS 1-4 & CFS 5-9 & $p$ value \\
& $N=285$ & $N=444$ & \\
\hline Female & $163(57.2 \%)$ & $291(65.5 \%)$ & 0.023 \\
Age (median, IQR) & $81(77-86)$ & $86(81-90)$ & $<0.001$ \\
ISS (median, IQR) & $16(9-21)$ & $10(9-17)$ & 0.00225 \\
GCS (mean, SD) & $14(1.31)$ & $14(1.11)$ & 0.5573 \\
Charleson comorbidity index (median, IQR) & $4(3-5)$ & $5(4-6)$ & $<0.001$ \\
Total length of stay (median, IQR) & $11(7-17)$ & $12(7-20)$ & 0.1498 \\
Trauma call received & $72(25.3 \%)$ & $58(13.1 \%)$ & $<0.001$ \\
Discharge to usual residence & $193(71.5 \%)$ & $192(49.6 \%)$ & $<0.001$ \\
Died & $15(5.3 \%)$ & $57(12.8 \%)$ & 0.001 \\
\hline
\end{tabular}

$I Q R$ interquartile range, ISS Injury Severity Score, GCS Glasgow Coma Scale

Table 2 Regression analyses demonstrating the impact of CFS 5-9 vs CFS 1-4 on trauma outcomes

\begin{tabular}{|c|c|c|c|c|c|c|c|c|}
\hline & $\begin{array}{l}\text { Mortality (OR, 95\% } \\
\text { CI) }\end{array}$ & $p$ value & $\begin{array}{l}\text { Length of stay }(\beta, \\
95 \% \mathrm{CI})\end{array}$ & $p$ value & $\begin{array}{l}\text { Received trauma } \\
\text { call (OR, } 95 \% \\
\text { CI) }\end{array}$ & $p$ value & $\begin{array}{l}\text { Discharged to usual } \\
\text { residence (OR, 95\% } \\
\text { CI) }\end{array}$ & $p$ value \\
\hline \multicolumn{9}{|l|}{ Unadjusted } \\
\hline CFS 5-9 & $2.65(1.47-4.78)$ & 0.001 & $2.17(0.25-4.09)$ & 0.027 & $0.44(0.30-0.65)$ & $<0.001$ & $0.39(0.28-0.55)$ & $<0.001$ \\
\hline \multicolumn{9}{|l|}{ Adjusted for } \\
\hline Age & $2.80(1.52-5.17)$ & 0.001 & $2.38(0.36-4.40)$ & 0.021 & $0.76(0.50-1.16)$ & 0.208 & $0.42(0.30-0.60)$ & $<0.001$ \\
\hline Sex & $2.85(1.57-5.16)$ & 0.001 & $2.34(0.41-4.27)$ & 0.018 & $0.47(0.32-0.69)$ & $<0.001$ & $0.41(0.29-0.57)$ & $<0.001$ \\
\hline $\begin{array}{l}\text { Charleson Comor- } \\
\text { bidity Index }\end{array}$ & $2.37(1.30-4.33)$ & 0.005 & $1.69(-0.29-3.68)$ & 0.096 & $0.61(0.40-0.92)$ & 0.018 & $0.39(0.28-0.56)$ & $<0 . .001$ \\
\hline ISS & $2.96(1.62-5.39)$ & $<0.001$ & $2.31(0.38-4.24)$ & 0.019 & $0.47(0.30-0.73)$ & 0.001 & $0.38(0.27-0.53)$ & $<.001$ \\
\hline $\begin{array}{l}\text { Trauma call } \\
\text { received }\end{array}$ & $2.81(1.54-5.11)$ & 0.001 & - & & - & & - & \\
\hline LOS & $2.77(1.53-5.01)$ & 0.001 & - & & - & & $0.41(0.29-0.57)$ & $<0.001$ \\
\hline $\begin{array}{l}\text { Adjusted for all } \\
\text { factors }\end{array}$ & $2.79(1.47-5.28)$ & 0.002 & $2.08(0.03-4.12)$ & 0.046 & $0.80(0.49-1.30)$ & 0.366 & $0.44(0.31-0.63)$ & $<0.001$ \\
\hline
\end{tabular}

there was no longer a significant association between CFS and trauma call status (OR 0.80, 95\% CI 0.49-1.30).

There were some highly significant differences between the characteristics of those who survived and the mortality group (Table 3). The mortality group had a higher ISS, was more likely to be CFS 5-9 and also had a higher Charleson comorbidity score.

\section{Discussion}

In most societies around the world, people are living both longer and more active lives. There has subsequently been a substantial increase in the amount of elderly trauma care required. Despite this, there is little evidence regarding assessment tools for predicting outcomes in this patient population. This study has shown that the use of CFS scores can help to predict outcomes in this patient group. We have demonstrated that frail geriatric trauma patients
Table 3 Characteristics of survival group vs mortality group

\begin{tabular}{lllr}
\hline & Alive & Dead & $p$ value \\
\hline ISS (median, IQR) & $10(9-18)$ & $16(9-25)$ & 0.001 \\
CFS 5-9 & $387(58.9 \%)$ & $57(79.2 \%)$ & $<0.001$ \\
Charleson comorbidity & $4(4-5)$ & $5(4-6)$ & 0.007 \\
score (median, IQR) & & & \\
\hline
\end{tabular}

are at much higher risk of in-hospital mortality, than those who are not. Having this understanding of potential outcomes in elderly trauma can assist clinicians when communicating with relatives and setting realistic expectations of care. Furthermore understanding the relationship between frailty and trauma will better allow the identification of those whom may benefit from appropriately invasive resuscitation and surgical management of survivable injuries. 
This is the largest UK study evaluating the relationship between CFS scores and outcomes in geriatric trauma. The possibility of misclassification of patients was reduced by prospective allocation of CFS scores. The entire cohort had been given CFS score during admission, and 52\% of these had been allocated by geriatrician as suggested by Rockwood and Mitinski [6]. The American College of Surgeons recommends frailty assessment in elderly trauma patients [7]. However, an ideal tool for this is controversial. There are numerous methods, all requiring varying amounts of time and information gathering [8]. In addition to this, some screening tools may be of more use in the research setting; others may be better suited for clinical use (either in acute or chronic setting) and some may be better applied when reviewing frailty and public health [9]. Despite the lack of consensus, we would advocate the use of CFS scoring in the acute or subacute phase for its ease of use, simplicity and demonstrable validity to identify those at risk of increased LOS, discharge to other care facility and mortality. CFS scoring is well suited for clinical screening, but perhaps a more in-depth tool used by geriatrician would be required for frailty assessment $[3,8,10]$. Frailty assessments involve a more holistic review of patient. When selecting, the instrument used to assess patients is important to consider the clinical context and who will be performing the test $[9,11]$.

In the setting of emergency surgical admissions, this score has been found to be a valid predictor of outcomes [12]. Our study results are in line with those of previously published works $[13,14]$, and contribute to the validation of CFS use in elderly trauma.

Analysis of the mortality group has shown that the most common mechanism of injury was a fall less than $2 \mathrm{~m}$ which is in keeping with TARN data [2], but contradicts previous work finding a fall greater than $2 \mathrm{~m}$ as a predictor of mortality [15]. The mortality groups were also less likely to have been trauma-called upon arrival to the emergency department, which is a factor of known significance $[2,7]$. Relatively trivial mechanism of injury, altered baseline mental state and reduced physiological response to injury all contribute to the under-triage in elderly trauma, potentially leading to under-resuscitation and further inadequate treatment during their stay $[2,7]$. It seems that frail trauma patients need to be treated with a much higher index of suspicion, despite having low-energy mechanism of injury, to ensure their injuries are discovered promptly and appropriate resuscitation can commence.

There are some limitations to this study. We have tried to account for confounding factors, such as comorbidity, by incorporating the Charleson Comorbidity Index scores into our analysis. This is a not an all-encompassing scale of comorbidities and other conditions which may be significant are not accounted for. The CFS is a subjective tool where clinicians may have differing opinions of a patient's frailty, leading to misclassification. However, this is a realistic reflection of clinical practice, which requires an overall clinical impression based on the information available at the time. There are also limitations associated with retrospective database reviews, such as missing or inaccurate data. In this instance, it was mainly patients who had not been allocated a CFS score during their admission which reduced the sample size. Attempts could have been made to retrospectively allocate CFS scores within this group; however, it may have introduced further bias. Other inaccuracies in the data have been limited through using the TARN submission data which will have been verified from multiple sources. There are also no follow-up data available for these patients, as 30-day or 1-year mortality may have been desirable.

Ultimately there is varying performance in the management of elderly trauma, despite there being advances in other aspects of trauma care. An American study benchmarking Trauma Centre performance has revealed a fivefold increase in the risk of death between low- and high-performing hospitals [16]. This discrepancy clearly highlights the need to identify opportunities for improved elderly trauma care which would logically start by identifying the most vulnerable. Contributing factors, such as under-triage, under-resuscitation, altered response to injury, pre-existing medical conditions and risk of in-hospital complications, all contribute to this varied performance and further research is needed to identify how these factors can be managed [16-19]. At our hospital, we have noted the rise in elderly trauma and have taken steps to improve care for this group of patients. This includes daily multidisciplinary team review of patients, involving physicians, nurses, dieticians, physiotherapists, occupational therapists and pharmacists. This is in addition to reviews from Elderly Care Physicians during the working week. This holistic approach to care is necessary when dealing with these patients given their complex medical needs.

In conclusion, this is the largest UK study assessing the impact of frailty in geriatric trauma using CFS scoring. We have shown that frail trauma patients have higher mortality rates and length of stay whilst being less likely to be traumacalled upon arrival. Frail patients are also more likely to be discharged to a nursing home or other care facility. Recent improvements in trauma care have not been reflected in elderly patients. We advocate further research into frailty and trauma, especially the shared management between Elderly Care physicians and the Major Trauma team.

Acknowledgements We would like to thank Jamie Corkill

Author contributions ATT: Study design, data collection, writing, SG: Literature search, study design, data collection, YN: Data collection, data interpretation, $\mathrm{CH}$ : Data analysis, data interpretation, writing, $\mathrm{AB}$ : Critical revision, writing. 
Funding No funding was acquired for this study.

\section{Declarations}

Conflict of interest There are no conflicts of interest.

\section{References}

1. Office of National Statistics, United Kingdom. Living longer: how our population is changing and why it matters. 2018. https://www. ons.gov.uk/peoplepopulationandcommunity/birthsdeathsandmarri ages/ageing/articles/livinglongerhowourpopulationischangingan dwhyitmatters/2018-08-13. Accessed 1 Mar 2021

2. Trauma Audit and Research Network. Major trauma in older people. 2017. https://www.tarn.ac.uk/content/downloads/3793/Major \%20Trauma\%20in\%20Older\%20People\%202017.pdf. Accessed 1 Mar 2021

3. Morley JE, Vellas B, van Kan GA, Anker SD, Bauer JM, Bernabei R, Cesari M, Chumlea WC, Doehner W, Evans J, Fried LP, Guralnik JM, Katz PR, Malmstrom TK, McCarter RJ, Gutierrez Robledo LM, Rockwood K, von Haehling S, Vandewoude MF, Walston J. Frailty consensus: a call to action. J Am Med Dir Assoc. 2013;14(6):392-7. https://doi.org/10.1016/j.jamda 2013.03.022.

4. Collard RM, Boter H, Schoevers RA, Oude Voshaar RC. Prevalence of frailty in community-dwelling older persons: a systematic review. J Am GeriatrSoc. 2012;60(8):1487-92. https://doi.org/10 .1111/j.1532-5415.2012.04054.x (Epub 2012 Aug 6).

5. Fit for frailty-developing, commissioning and managing services for people living with frailty in community settings-a report from the British Geriatrics Society and the Royal College of General Practitioners. 2015. https://www.bgs.org.uk/sites/default/files /content/resources/files/2018-05-23/fff2_full.pdf. Accessed 1 Mar 2021

6. Rockwood K, Song X, MacKnight C, Bergman H, Hogan DB, McDowell I, Mitnitski A. A global clinical measure of fitness and frailty in elderly people. CMAJ. 2005;173(5):489-95. https://doi. org/10.1503/cmaj.050051.

7. American College of Surgeons: Trauma Quality Improvement Programme. Geriatric trauma management guidelines. 2013. https ://www.facs.org/-/media/files/quality-programs/trauma/tqip/geria tric_guidelines.ashx।. Accessed 1 Mar 2021

8. Dent E, Kowal P, Hoogendijk EO. Frailty measurement in research and clinical practice: a review. Eur J Intern Med. 2016;31:3-10. https://doi.org/10.1016/j.ejim.2016.03.007 (Epub 2016 Mar 31).

9. Gilardi F, Capanna A, Ferraro M, Scarcella P, Marazzi MC, Palombi L, Liotta G. Frailty screening and assessment tools: a review of characteristics and use in Public Health. Annali di
IgieneMedicinaPreventiva e di Comunita. 2018;30(2):128-39. https://doi.org/10.7416/ai.2018.2204.

10. Joseph B, Pandit V, Zangbar B, Kulvatunyou N, Hashmi A, Green DJ, O'Keeffe T, Tang A, Vercruysse G, Fain MJ, Friese RS, Rhee P. Superiority of frailty over age in predicting outcomes among geriatric trauma patients: a prospective analysis. JAMA Surg. 2014;149(8):766-72. https://doi.org/10.1001/jamasurg.2014.296.

11. Buta BJ, Walston JD, Godino JG, Park M, Kalyani RR, Xue QL, Bandeen-Roche K, Varadhan R. Frailty assessment instruments: systematic characterization of the uses and contexts of highlycited instruments. Ageing Res Rev. 2016;26:53-61. https://doi. org/10.1016/j.arr.2015.12.003.

12. Parmar KL, Law J, Carter B, Hewitt J, Boyle JM, Casey P, Maitra I, Farrell IS, Pearce L, Moug SJ, ELF Study Group. Frailty in older patients undergoing emergency laparotomy: results from the UK observational Emergency Laparotomy and Frailty (ELF) study. Ann Surg. 2019. https://doi.org/10.1097/SLA.0000000000 003402 (Epub ahead of print).

13. Cheung A, Haas B, Ringer TJ, McFarlan A, Wong CL. Canadian study of health and aging Clinical Frailty Scale: does it predict adverse outcomes among geriatric trauma patients? J Am CollSurg. 2017;225(5):658-665.e3. https://doi.org/10.1016/j.jamco 1lsurg.2017.08.008 (Epub 2017 Sep 6).

14. Curtis E, Romanowski K, Sen S, Hill A, Cocanour C. Frailty score on admission predicts mortality and discharge disposition in elderly trauma patients over the age of $65 \mathrm{y}$. J Surg Res. 2018;230:13-9. https://doi.org/10.1016/j.jss.2018.04.017 (Epub 2018 May 12).

15. Pecheva M, Phillips M, Hull P, Carrothers AOR, Queally JM. The impact of frailty in major trauma in older patients. Injury. 2020;51(7):1536-42. https://doi.org/10.1016/j.injury.2020.04.045 (Epub 2020 May 12).

16. Haas B, Gomez D, Xiong W, Ahmed N, Nathens AB. External benchmarking of trauma center performance: have we forgotten our elders? Ann Surg. 2011;253(1):144-50. https://doi. org/10.1097/SLA.0b013e3181f9be97.

17. Jacobs DG, Plaisier BR, Barie PS, Hammond JS, Holevar MR, Sinclair KE, Scalea TM, Wahl W, EAST Practice Management Guidelines Work Group. Practice management guidelines for geriatric trauma: the EAST Practice Management Guidelines Work Group. J Trauma. 2003;54(2):391-416. https://doi. org/10.1097/01.TA.0000042015.54022.BE.

18. Champion HR, Copes WS, Buyer D, Flanagan ME, Bain L, Sacco WJ. Major trauma in geriatric patients. Am J Public Health. 1989;79(9):1278-82. https://doi.org/10.2105/ajph.79.9.1278.

19. Lin HS, Watts JN, Peel NM, Hubbard RE. Frailty and post-operative outcomes in older surgical patients: a systematic review. BMC Geriatr. 2016;16(1):157. https://doi.org/10.1186/s1287 7-016-0329-8. 